\title{
Emerging bioinspired polymers: glycopolypeptides
}

Cite this: Soft Matter, 2014, 10, 4228

Received 14th February 2014 Accepted 26th March 2014

DOI: $10.1039 / \mathrm{c} 4 \mathrm{sm} 00352 \mathrm{~g}$

www.rsc.org/softmatter

\section{Introduction}

Bioinspired or biohybrid polymers are an emerging class of materials that are designed to combine advantageous features of synthetic polymers, such as solubility, processability, and scalability, with those of biological entities like chirality, selective recognition, or signaling., ${ }^{1,2}$ Recent efforts have been focused on sugar-containing polymers, including carbohydratesynthetic copolymers, also block copolymers, and polymers with pendant carbohydrates - the latter being usually referred to as glycopolymers. ${ }^{3}$

Since the first reported examples in the early $1960 \mathrm{~s},{ }^{4-6}$ many different glycopolymers have been produced by free or

Max Planck Institute of Colloids and Interfaces, Department of Colloid Chemistry, Research Campus Golm, 14424 Potsdam, Germany. E-mail: Helmut.Schlaad@ mpikg.mpg.de

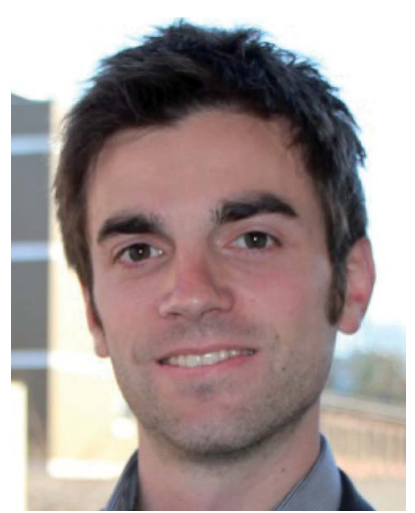

Kai-Steffen Krannig studied chemistry at the University of Marburg, Germany, and the University of British Columbia in Vancouver, Canada. He received his Diploma in 2011. He then joined the group of Helmut Schlaad at the Max Planck Institute of Colloids and Interfaces in Potsdam, Germany, to pursue a doctoral degree which he obtained with honors (summa cum laude) in 2013. After a post-doctoral period with Markus Antonietti, he moved on to Evonik Industries in Marl, Germany. controlled radical polymerization of vinyl monomers and ringopening metathesis polymerization of norbornenes..$^{7-9}$ All backbones are carbon chains, which have no other functions than to connect the carbohydrate residues and to serve as spacer units. Polypeptides, on the other hand, have the intriguing ability to fold into secondary, tertiary, and higher order structures due to multiple non-covalent interaction amino acid units and are inherently biocompatible and biodegradable. Naturally occurring glycosylated peptides and proteins display a wide range of biological functions including anti-freezing, ${ }^{10}$ mediation of recognition events, proliferation of cells, and inflammatory reactions. ${ }^{11-14}$ Hence their synthetic counterparts are also expected to have great potential as biomedical materials (e.g., drug carriers or scaffolds for tissue repair) and to serve as versatile tools for probing carbohydrateprotein interactions. ${ }^{15,16}$ Synthetic glycopeptides have been known for 20 years, ${ }^{17}$ nevertheless the production of

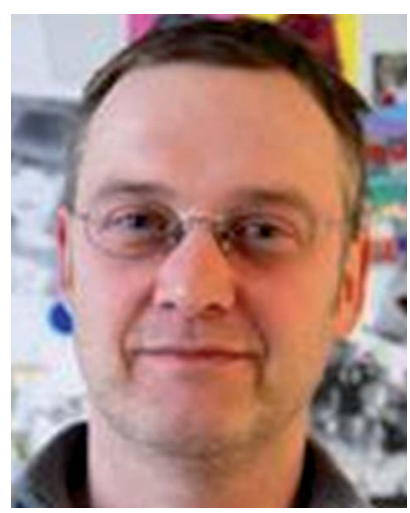

Helmut Schlaad studied chemistry at the University of Mainz, Germany, and earned a doctoral degree in Physical Chemistry, under the supervision of Axel $\mathrm{H}$. E. Müller in 1997. After a one year post-doctoral fellowship with Rudolf Faust at the University of Massachusetts in Lowell, USA, he moved to the Max Planck Institute of Colloids and Interfaces in Potsdam, Germany. He finished habilitation, mentored by Markus Antonietti, and became a senior scientist in 2004. He was recently called to join the University of Potsdam as a Professor (W2) of Polymer Chemistry. His research interests are directed towards polymer synthesis, smart functional materials, and bioinspired structures based on synthetic and biohybrid polymers. 
well-defined glycopolypeptides in larger quantities is still a challenging task and the understanding of their hierarchical assembly, as an indispensable condition to a smart design of advanced functional materials, is in its infancy.

This article presents a selection of very recent advances in the field of glycopolypeptides, covering the synthesis, stimuliresponsive secondary structures and self-assemblies, and biological applications. Comprehensive and more detailed information can be found in a number of excellent reviews and perspectives that have been published in the last year. ${ }^{18-22}$

\section{Synthesis of glycopolypeptides}

Two different pathways are applied to produce glycopolypeptides (Fig. 1). The first route involves the synthesis of a glycosylated $\mathrm{N}$-carboxyanhydride, referred to as glyco-NCA, which then is polymerized in a controlled manner to yield high molar mass glycopolypeptides. The main issue or problem is the synthesis and purification of glyco-NCA, which requires a high level of synthesis expertise. The second route is the synthesis of a functionalizable polypeptide precursor chain, which is subjected to post-polymerization modification to introduce the carbohydrate residues. The synthesis is modular and much less elaborate than the first route without compromising the degree of glycosylation, however, the glycopolypeptides obtained by modification of polypeptides have considerably lower molar masses than the ones produced by glyco-NCA polymerization.

\subsection{Synthesis and polymerization of glyco-NCAs}

Okada et al. described the first synthesis of a glycopeptide through amine-initiated ring-opening polymerization of glycoNCA, namely $O$-(tetra- $O$-acetyl- $\beta$-D-glucopyranosyl)-L-serine. ${ }^{17}$ This glyco-NCA was obtained by coupling tetra-O-acetylated glucose to serine, as described by Rüde $e t$ al. ${ }^{23}$ and subsequent

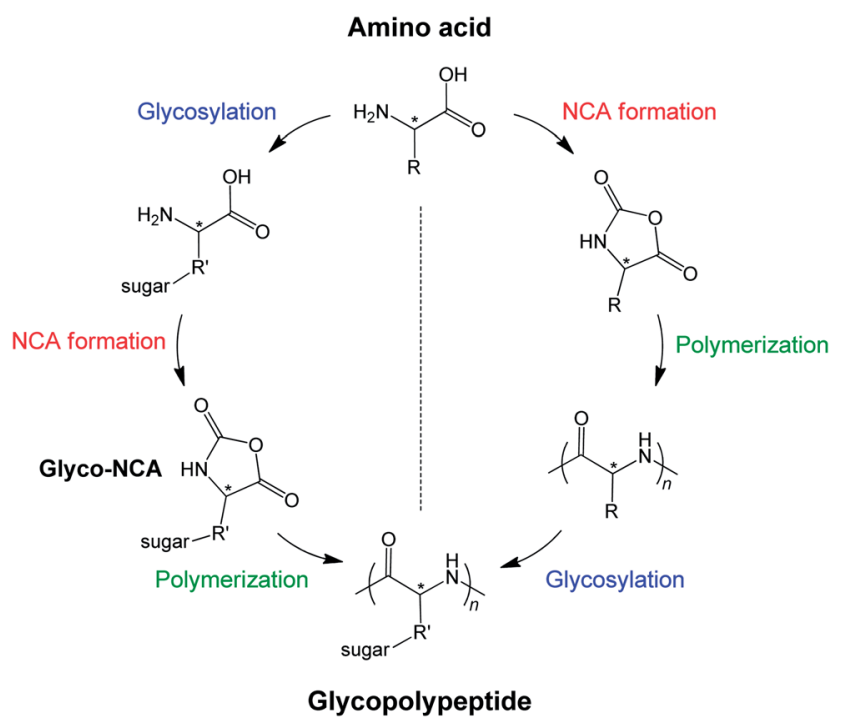

Fig. 1 Synthetic pathways towards glycopolypeptides: (1) synthesis and polymerization of glyco-NCA (left) and (2) synthesis of a functionalizable polypeptide and subsequent glycosylation (right). reaction of the amino acid with phosgene. Application of primary amine initiators produced well-defined glycopeptides with number-average molar masses $\left(M_{\mathrm{n}}\right)$ of about $10 \mathrm{~kg} \mathrm{~mol}^{-1}$ and low dispersity, $M_{\mathrm{w}} / M_{\mathrm{n}} \approx 1.1$ (ratio of weight- over numberaverage molar mass). The living character of the polymerization was demonstrated by the addition of a second monomer, alanine-NCA, to yield block copolypeptides. ${ }^{17}$

The synthesis of higher molar mass glycopolypeptides requires glyco-NCAs in high purity, which is made difficult by their high reactivity. Recently, in 2010, Deming et al. succeeded in the chromatographic purification of glycosylated Llysine-NCAs and were able to achieve high molar mass polypeptides $\left(M_{\mathrm{n}} \approx 160 \mathrm{~kg} \mathrm{~mol}{ }^{-1}\right)$ with low dispersity. ${ }^{24,25}$ Peracetylated sugars (glucose, galactose, or mannose) were coupled to L-lysine in a four-step procedure and the amino acid transferred into NCA by treatment with dichloro(methoxy) methane. The glyco-NCAs were afterwards purified by anhydrous flash chromatography. Polymerization using a transition metal catalyst, $\left(\mathrm{PMe}_{3}\right)_{4} \mathrm{Co}$, yielded homo and block copolypeptides, which after deacetylation could be readily dispersed in water.

Sen Gupta et al. reported another procedure towards glycoNCAs based on L-lysine. ${ }^{26-28}$ A protected lysine, CbzLys(Boc)OBn $(\mathrm{Cbz}=$ benzyloxycarbonyl, Boc $=$ tert-butyloxycarbonyl, $\mathrm{Bn}=$ benzyl), was coupled with the 1,2-orthoesters of either glucose or mannose in the presence of a gold catalyst. After removal of the acid protecting group by hydrogenation, the amino acid was converted into the NCA by the Fuchs-Farthing method using triphosgene (and $\alpha$-pinene as hydrogen chloride scavenger). These lysine derived glyco-NCAs were purified by conventional recrystallization. The polymerization reactions of glyco-NCAs, initiated by 1-hexylamine, were only successful in the presence of the 1,8-bis(dimethylamino)naphthalene "proton sponge" neutralizing residual acid impurities (however, the exact role of the base is not fully understood yet). The obtained homo- and block copolypeptides exhibited low dispersity but usually much higher than expected molar masses.

Wenz et al. synthesized a sugar-appended lysine-NCA in a three-step procedure by glycosylation of 2-(2-isothiocyanatoethoxy)ethanol with 1-trichloroacetimidate-2,3,4,6tetra-O-acetyl-glycopyranose and subsequent coupling of the isothiocyanate group to the $\varepsilon$-amino group of $\mathrm{Cbz}$-lysine. ${ }^{29}$ Treatment with dichloromethyl(methoxy)methane yielded NCA, which was then copolymerized with PEGylated lysine- and $\varepsilon$ TFA-lysine-NCA using either triethylamine or the (bipy) $\mathrm{Ni}(\mathrm{COD})$ catalyst.

Glyco-NCAs were also obtained from L-cysteine by photochemical addition of the amino acid thiol to 1-allyl-2,3,4,6-tetra$O$-acetyl- $\alpha$-D-glucose or -galactose (employing 2,2-dimethoxy-2phenylacetophenone as a photoinitiator and UV at $365 \mathrm{~nm}$ ) and subsequent reaction with dichloro(methoxy)methane. ${ }^{30}$ Purification was done by either column chromatography or aqueous workup and repeated precipitation. The glyco-NCAs could be polymerized efficiently with the $\left(\mathrm{PMe}_{3}\right)_{4} \mathrm{Co}$ initiator in tetrahydrofuran at room temperature to yield protected glycopolypeptides with molar masses $\left(M_{\mathrm{n}}\right)$ up to $90 \mathrm{~kg} \mathrm{~mol}^{-1}$ and low dispersity $(<1.1)$. 
The syntheses of other $O$ - and $S$-linked glyco-NCAs based on L-threonine, $\mathrm{L}$-serine, and $\mathrm{L}$-cysteine have also been described. ${ }^{31}$ The acetobromo derivatives of glucose, galactose, or lactose were coupled to the respective amino acids in the presence of iodine as a Lewis acid promoter and subsequently reacted to the NCA using triphosgene and $\alpha$-pinene in ethyl acetate. Polymerization reactions of such NCAs have not been reported yet.

Although a variety of glycosylated amino acid NCAs have been successfully prepared applying sophisticated organic chemistry protocols, the tedious multi-step synthesis and purification procedures and often low yields are severe drawbacks of this approach.

\subsection{Post-polymerization glycosylation of synthetic polypeptides}

Several approaches for the modification of already existing polypeptides have been described since 2010, attributable to the popularity of "click" chemistry. ${ }^{32-34}$ In particular, the coppercatalyzed azide-alkyne $[3+2]$ cycloaddition (CuAAC) became a broadly applied technique in not only the synthesis of glycosylated monomers but also in the post-polymerization functionalization of polymers. ${ }^{7,35}$

Zhang et al. synthesized a $\gamma$-chloropropyl-L-glutamate-NCA by the monoesterification of glutamic acid with 3-chloropropyl alcohol in the presence of chlorotrimethylsilane and subsequent treatment with triphosgene, and polymerized it using a hexamethyldisilazane initiator. ${ }^{36,37}$ The obtained polypeptides $\left(M_{\mathrm{n}}=5-28 \mathrm{~kg} \mathrm{~mol}{ }^{-1}\right.$, dispersity $\left.\approx 1.2\right)$ were reacted with sodium azide to give poly( $\gamma$-azidopropyl-L-glutamate), which allowed for quantitative addition of 1-propargyl- $\alpha$-D-mannose via CuAAC. Alternatively, poly $(\gamma$-propargyl-L-glutamate) was prepared by primary amine initiated ring-opening polymerization of the corresponding amino acid-NCA and then reacted with sugar-azides applying CuAAC. ${ }^{38}$

The CuAAC approach was also applied to polypropargylglycine, avoiding any hydrolytically unstable ester linkage in the polypeptide side chains.$^{39-41}$ Polypropargylglycine homopolymers and statistical and block copolymers (with $\gamma$ benzyl-L-glutamate) were synthesized by primary amine initiated ring-opening polymerization of propargylglycine-NCA (readily obtained by the reaction of the commercial amino acid with triphosgene) and were glycosylated with 1-azido- $\beta$-Dgalactose in the presence of triethylamine and catalytic amounts of $\left(\mathrm{PPh}_{3}\right)_{3} \mathrm{CuBr}$ in DMSO solution.

Polyallylglycines (and the related polydepsipeptides) have been introduced as another clickable polypeptide by thiol-ene chemistry, connecting functional groups to the polypeptide chains with a thioether linker (as in methionine). ${ }^{42,43}$ Polyallylglycines, as well as polypropargylglycines, are poorly soluble in common organic solvents or water, making the synthesis of higher molar mass polypeptides and quantitative modification difficult or even impossible. Polyallylglycine with rather a low molar mass of $M_{\mathrm{n}}<2 \mathrm{~kg} \mathrm{~mol}^{-1}$ could only be modified quantitatively with 1 -thio- $\beta$-D-glucopyranose, to yield poly[(3- $\left(\beta-\right.$ D- $^{-}$ glucopyranosyl)thio)propylglycine], when the reaction was run in trifluoroacetic acid (TFA) over two days. This is not ideal because TFA is a hazardous solvent and may not be applicable to more sensitive biological substrates. Interestingly, low molar mass poly( $N$-allyl glycine) $\left(M_{\mathrm{n}}<2 \mathrm{~kg} \mathrm{~mol}{ }^{-1}\right)$, which is the peptoid structural isomer to polyallylglycine, is soluble in water and could be readily converted into a glycopolypeptoid by photoaddition of 1-thio- $\beta$-D-glucopyranose within less than a day. ${ }^{\mathbf{4 4}}$

Recently, statistical copolymers of allyl- or propargylglycine and $\gamma$-benzyl-L-glutamate, exhibiting much better solubility, have been synthesized and glycosylated in organic media or in aqueous media (acetate buffer) after debenzylation of the glutamate units. ${ }^{45,46}$ The single addition of the thio-sugar to allylglycine units was found to proceed smoothly and quantitatively, but double addition of sugar to propargylglycine proved to be more difficult (only achieving $<1.5$ equivalents). Importantly, the modification reaction occurs in water using unprotected sugars and may well be applied to other thiol bearing biological materials.

Functional cationic polypeptides including glycopolypeptides have been synthesized by the quantitative alkylation of poly(L-methionine)s with alkyl halides or triflates. ${ }^{47}$ For instance, methionine alkylation with 2-(2,3,4,6-tetra- $O$-acetyl- $\alpha$ D-galactopyranosyl)ethyl triflate in a solvent mixture of dichloromethane-acetonitrile at $20{ }^{\circ} \mathrm{C}$ produced the corresponding $S$-linked glycopolypeptide in $97 \%$ yield. Interestingly, alkylation of the methionine units is reversible and dealkylation can be induced when desired. ${ }^{48}$

$N$-Linked glycopolypeptides were obtained by condensation of $\beta$-glycosylamines to poly(L-glutamic acid $)^{49}$ or by aqueous amide coupling of D-glucosamine hydrochloride to poly(L-glutamic acid) $\left(M_{\mathrm{n}}=9-180 \mathrm{~kg} \mathrm{~mol}^{-1}\right)$ using 4-(4,6-dimethoxy-1,3,5triazin-2-yl)-4-methylmorpholinium chloride (DMT-MM) as a coupling agent. ${ }^{50}$ The latter reaction was performed without any organic solvents, additives, or buffers, and the degree of substitution could be controlled by the molar ratio of DMT-MM to glutamate to about $80 \%$. A similar approach has been used for glycosylation of a protein sequence containing glutamic acid residues with $\beta$-D-galactosamine using $N, N, N^{\prime}, N^{\prime}$-tetramethyl-O(1H-benzotriazol-1-yl)uranium hexafluorophosphate (HBTU) as a coupling agent under basic conditions. ${ }^{51}$ Alternatively, poly(Llysine) was glycosylated with D-gluconolactone in the presence of diisopropylethylamine in DMSO-water at room temperature. ${ }^{52}$

The post-polymerization glycosylation approach appears to be a good alternative to glyco-NCA polymerization, though there are still limitations, applying to some but not all strategies, regarding molar mass and degree of glycosylation. However, the polypeptide precursors are modular platform materials that can be decorated with multiple substrates to yield heterofunctional glycopolypeptides for a variety of tasks.

\section{Aqueous solution and self- assembly behavior}

A key feature of polypeptides is their intrinsic ability to form distinct secondary and higher-order structures, which is usually not observed for conventional synthetic polymers (e.g., polyolefines, polyacrylates, or polyesters). Exceptions being materials such as polyamides (nylons) which form sheet-like 
superstructures through intermolecular hydrogen bonding, ultimately leading to properties such as high toughness. Synthetic polypeptide structures can reach a higher complexity and sophistication and, in addition, adapt to changes in environmental conditions (temperature, solution $\mathrm{pH}$, ionic strength, etc.) or respond to other kinds of chemical, physical, or biological stimuli, making them promising building blocks for bioinspired or biomimetic structure formation.

\subsection{Stimuli-responsive behavior}

Temperature. Glycosylated poly(L-lysine)s were found to adopt an $\alpha$-helical conformation in aqueous solution at neutral $\mathrm{pH} .^{25}$ Surprisingly, the preferred conformation of the poly( $\mathrm{L}^{-}$ lysine) backbone was not disturbed by the presence of the bulky sugar groups in the side chains. Upon increasing the temperature from $4{ }^{\circ} \mathrm{C}$ to $90{ }^{\circ} \mathrm{C}$ the helicity of the glycopolypeptide chains $\left(M_{\mathrm{n}}=90 \mathrm{~kg} \mathrm{~mol}^{-1}\right)$ decreased gradually from $100 \%$ to about $40 \%$, attributable to the disruption of hydrogen bonding through interactions with water molecules. ${ }^{53}$ Helicity was only found to be about $80 \%$, meaning that chain conformation is a disordered and not perfect $\alpha$-helix, in the biological relevant temperature range of $36-42{ }^{\circ} \mathrm{C}$.

Similar thermo-responsive behavior could also be observed for poly[L-glutamate-stat-(3-( $\beta$-D-glucopyranosyl)thio)propylglycine] $\left(M_{\mathrm{n}} \approx 15 \mathrm{~kg} \mathrm{~mol}{ }^{-1}\right) .{ }^{54}$ Absolute helicities were determined to be $60-70 \%$ (maximum value for $\mathrm{pH} 3.5$ ) at $37^{\circ} \mathrm{C}$, roughly only $10 \%$ less to room temperature. The helicity further decreases down to about $40 \%$ upon increasing the temperature to $90{ }^{\circ} \mathrm{C}$. The original secondary structure (helicity) could be fully retained after cooling back to room temperature, indicating that the conformational transition (or denaturation) is a fully reversible process.

Both glycopolypeptides undergo temperature-induced helixcoil transition, though the observed changes in helicity are rather minor in the relevant temperature window of biomedical applications.

Solution pH. Poly(L-glutamate) and poly(L-lysine) are the most frequently studied peptide-based polyelectrolytes undergoing helix-coil transition in complementary $\mathrm{pH}$ regions. ${ }^{55,56}$ The chains adopt helical conformations at $\mathrm{pH}<\mathrm{pK}_{\mathrm{a}}$ and $\mathrm{pH}>\mathrm{pK}_{\mathrm{a}}$, respectively, else random coil conformations, depending on the degree of ionization of the side chains. Decreasing ionization and electrostatic repulsion promotes folding of the chains into helices and disfavors a random coil conformation. This folding into helices is also accompanied by a decrease in hydrophilicity ultimately leading to precipitation from aqueous solution. Very few examples exist where ionic polypeptides, including glycopolypeptides, produce stable helices in water. ${ }^{\mathbf{4 5 , 5 0 , 5 4 , 5 7}}$

Poly[L-glutamate-stat-( $N$-glucopyranosyl)-L-glutamine]s adopt random coil conformation at neutral $\mathrm{pH}$ and helical conformation at acidic pH 4.2 (in $0.01 \mathrm{M}$ acetate buffer).$^{50}$ The helicity was found to decrease with increasing fraction of glycosylated glutamine units, and the helicity of a polypeptide chain with $78 \%$ glutamine units was just about $10 \%$. Only the copolypeptides with $63 \%$ or less glycosylated units were able to undergo a pH-responsive and reversible helix-to-coil transition.
Poly[L-glutamate-stat-(3-( $\beta$-D-glucopyranosyl)thio)propylglycine]s exhibited similar $\mathrm{pH}$-responsive behavior including reversible helix-coil transition without $\beta$-sheet formation. ${ }^{54}$ Helices remained stable and soluble in water or saline solution down to $\mathrm{pH} 3.5 .^{45}$ The maximum helicity was about $78 \%$ at $\mathrm{pH}$ 4.0, irrespective of the composition of the polypeptide chain, and was even higher for a poly(L-glutamate) homopolymer (71\%) of the same chain length.

Redox. Materials responding to reductive or oxidative environments are interesting for use in biomedical applications. ${ }^{58}$ Sulfur-containing polymers, and in particular cysteine- and methionine-based polypeptides, are candidates for redox chemistry (i.e., thiol/disulfide and thioether/sulfoxide/sulfone). The thiol/disulfide redox system can be applied for reversible crosslinking or functionalization, whereas with the thioether/ sulfoxide/sulfone system it is possible to alter the polarity and thus hydrophilicity of the polypeptide chains.

Poly[ $S$-(1-propyl- $\alpha$-D-galactopyranosyl)-L-cysteine $]$ adopts an $\alpha$-helical conformation in aqueous solution, which is destabilized and transformed into a random coil after oxidation of the thioether to sulfone (reduction pathway not reported yet). ${ }^{30}$ Mild oxidation of the linker to the sulfoxide, on the other hand, did not cause destabilization of the $\alpha$-helix. It is thought that the increased polarity and interaction of the sulfone with water lead to disruption of hydrophobic packing of side chains and increased steric crowding around the polypeptide backbone, thereby destabilizing the $\alpha$-helix. Interestingly, glycosylated poly(L-homocysteine)s, which carry an additional methylene group between the backbone and sulfur, adopt helical conformations irrespective of the oxidation state. Poly[L-(3-( $\beta$-D-glucopyranosyl)thio)propylglycine] with a C3 spacer, however, prefers a disordered random coil conformation. ${ }^{54}$ Apparently, the spacing between sulfur and the backbone is a sensitive balance with a tremendous impact on the chain folding.

Poly[L-glutamate-stat-(3-( $\beta$-D-glucopyranosyl)thio)propylglycine] with a thioether linker in the side chain is also expected to respond to a redox stimulus (in addition to temperature and $\mathrm{pH}$, see above), though there are no current reports.

\subsection{Self-assemblies in aqueous solution}

Polypeptide scaffolds can produce a large variety of complex structures, which is controlled by the chemistry and type of the side chain functional groups, i.e., primary structure. Sophisticated primary structures as in biological polypeptides or proteins have not yet been realized for synthetic polypeptides (via NCA polymerization), only simple codes like homopolymers or copolymers (statistical, alternating, gradient, or block structures; two or more comonomers). Polypeptides have been used in peptide-peptide or polymer-peptide (biohybrid) block copolymers to produce self-assemblies in water, particularly micelles, vesicles, and hydrogels, designated for use in biomedical applications. $^{2}$ Until now, very few studies on the self-assembly behavior of synthetic glycopolypeptides have been reported.

Lecommandoux, Heise et al. produced a variety of different morphologies of amphiphilic poly $(\gamma$-benzyl-L-glutamate)-block-(glyco-polypropargylglycine) copolypeptides by the 
nanoprecipitation technique (Fig. 2). ${ }^{40}$ Addition of water to a solution of the glycopolypeptide (hydrophilic weight fraction $>50 \%$ ) in dimethylsulfoxide (DMSO) and subsequent removal of the organic solvent by dialysis resulted in the formation of spherical and worm-like structures in water. Changing the order of addition of the solutions produced exclusively spherical structures such as micelles and small vesicles with an average diameter of $<100 \mathrm{~nm}$ and a 5-10 nm thick membrane. All observed morphologies are non-equilibrium structures and resulted from kinetic trapping induced by the rigidity of the hydrophobic poly $(\gamma$-benzyl-L-glutamate) segment.

Deming et al. described the aqueous self-assembly of two amphiphilic poly(L-leucine)-block-glyopolypeptide samples, where the hydrophobic segment had $\alpha$-helical conformation (poly(L-leucine)) and the hydrophilic glycosylated segment adopted either $\alpha$-helical (poly( $\alpha$-D-galactopyranosyl-L-lysine)) or random coil conformation (poly $(\alpha$-D-galactopyranosyl-L-cysteine sulfone)) (Fig. 3). ${ }^{59}$ The all-helical sample was found to assemble into micron sized irregular aggregates and plate-like objects, due to the rigidity of the hydrophilic domains. Poly(L-leucine)block-poly( $\alpha$-D-galactopyranosyl-L-cysteine sulfone) with a more flexible hydrophilic segment instead formed vesicles of about $100 \mathrm{~nm}$ in diameter (Fig. 3).
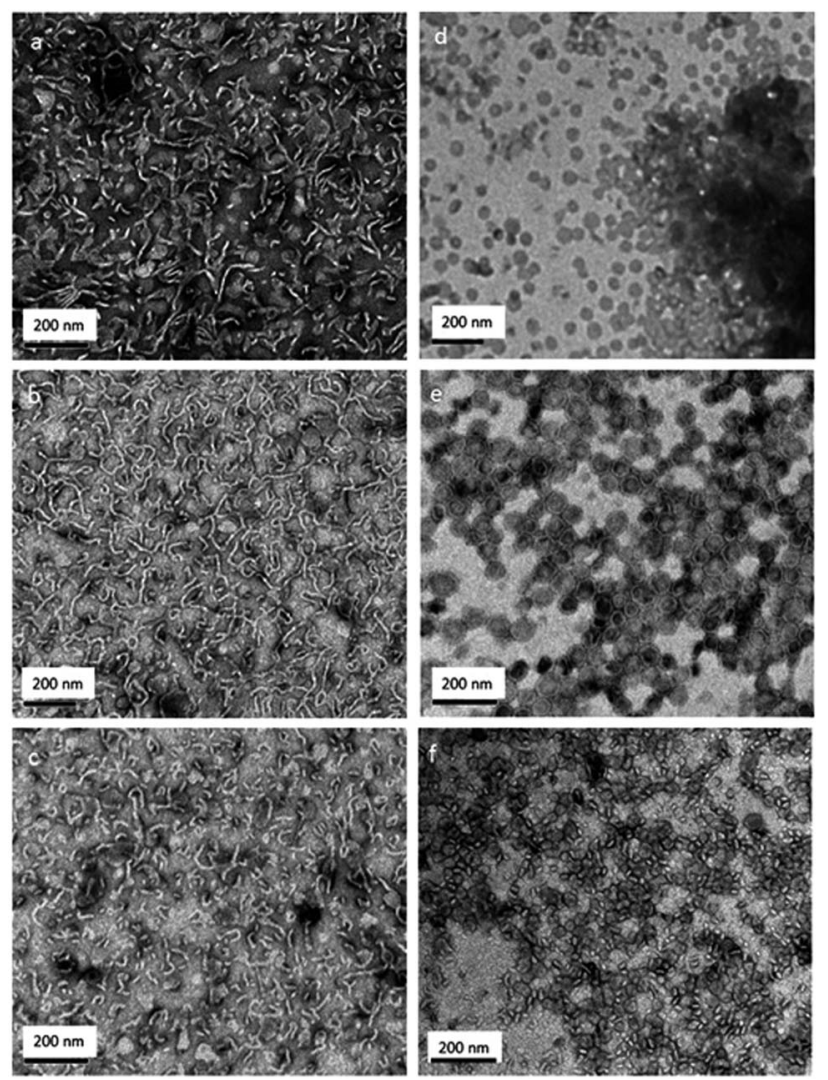

Fig. 2 Transmission electron micrographs of poly $(\gamma$-benzyl-L-glutamate)-block-(glyco-polypropargylglycine) (PBLG-b-PGG) samples obtained by fast addition of $(a-c)$ water in DMSO and $(d-f) D M S O$ in water: (a and d) PBLG $G_{20}-b-P G_{18}$; (b and e) PBLG ${ }_{20}-b-P G_{25}$; (c and f) $P B G_{20}-b-P_{32}$ (subscripts denote the average number of repeat units). Reprinted with permission from ref. 40, copyright (2012) American Chemical Society.
Sen Gupta described the self-assembly of amphiphilic glycopolypeptide-dendron block copolymers of glycosylated poly(L-lysine) attached to an aromatic dendron of generation 1 or 2 (see Fig. 4, top). ${ }^{60}$ Depending on the length of the glycopolypeptide (average number of repeat units: 14, 16, or 28) and dendron generation ( 1 or 2 ), either an organogel was obtained in DMSO or nanorods and micellar aggregates in aqueous solution (Fig. 4). The rod-like fibers, which exhibited a compartmentalized structure, were $50 \mathrm{~nm}$ in width and hundreds of nanometers in length, the length increases with decreasing mole fraction of the glycopolypeptide. The self-assembly behavior was explained by the cooperative effects of chain segregation and $\pi-\pi$-stacking of the dendrons. Interestingly, nanorods could only be observed for the L- but not for DL-glycopolypeptide-dendron copolymers, suggesting a correlation between the glycopolypeptide secondary structure and the selfassembly.

Amphiphilic $\mathrm{AB}_{n}$ heteroarm star ("tree-like") copolypeptides based on poly( $\gamma$-benzyl-L-glutamate)-block-(oligosaccharide-polypropargylglycine) (A = poly $(\gamma$-benzyl-L-glutamate), $\mathrm{B}=$ dextran or hyaluronan, $n=5$, average number of arms) were found to form very small spherical assemblies with sizes below $50 \mathrm{~nm}$ and low dispersity, by direct dissolution in water. ${ }^{41}$ Here, spherical particles with high curvature are formed due to the large hydrophilic weight fraction of $>60 \%$, despite the intrinsic rigidity of the hydrophobic poly( $\gamma$-benzyl-L-glutamate).

\section{Biological applications}

\section{Lectin binding}

Recognition is the first step in numerous biological processes based on cell-cell interactions. In many cases these recognition events are based on specific carbohydrate-protein (lectin) interactions that occur on the surface of cells. ${ }^{61}$ Lectins bind specifically but weakly to carbohydrates, and the binding can be enhanced by combining several carbohydrates in the same molecule (oligosaccharide or glycopolymer) or aggregate allowing for multiple binding events at the same time (cluster glycoside effect). ${ }^{7,35}$ However, the mechanisms and influence of the ligand structure on the multivalent binding interactions and the glyco code were largely unknown yet.

Several studies have been reported to demonstrate that the synthetic glycopolypeptides can indeed recognize and specifically interact with lectins, usually by turbidity assays, ${ }^{27,28,38,40,45,49,59}$ surface plasmon resonance (SPR), ${ }^{62}$ and isothermal titration calorimetry (ITC). ${ }^{27}$ The lectins used were usually Concanavalin A (ConA) for the selective binding of glucose and mannose and Ricinus communis agglutinin $\left(\mathrm{RCA}_{120}\right)$ for galactose. Interestingly, it has been found evidence that the degree of glycosylation (known as epitope density) ${ }^{\mathbf{1 5}}$ affects the kinetics of lectin binding ${ }^{38}$ and that the secondary structure of the glycopolypeptide ( $\alpha$-helix or random coil) has no impact on the binding affinity. ${ }^{27}$

Wenz et al. showed that galactosylated fluorescent poly(Llysine)s could be specifically incorporated in human $\mathrm{T}$ lymphocytes at $37{ }^{\circ} \mathrm{C}$, hence are potentially useful for selective staining of cells for targeted drug delivery. ${ }^{29}$ 


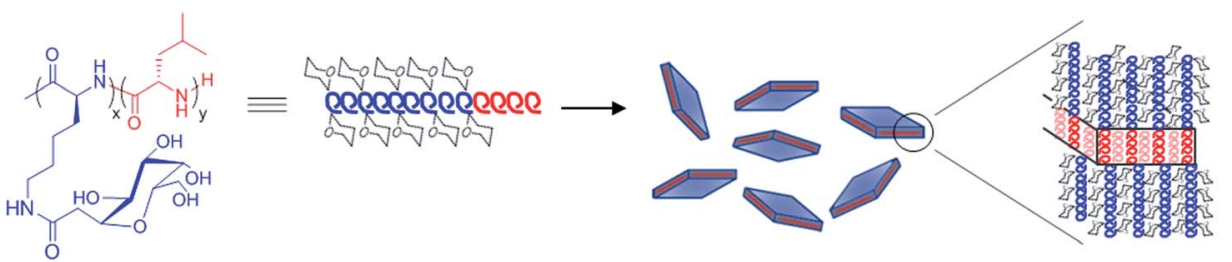

$(\alpha-g a l-K)_{65} L_{20}$

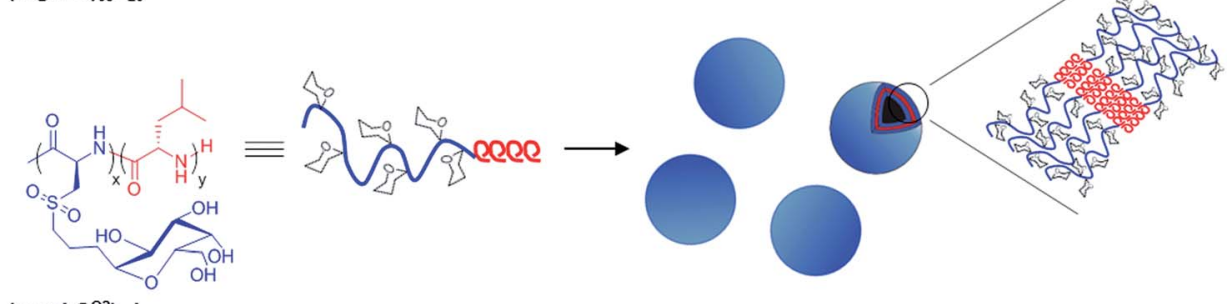

$\left(\alpha-g a l-\mathrm{C}^{\mathrm{O}}\right)_{65} \mathrm{~L}_{20}$

Fig. 3 Structures of amphiphilic glycosylated diblock copolypeptides and observed self-assemblies in water. Reprinted with permission from ref. 59, copyright (2013) Royal Society of Chemistry.
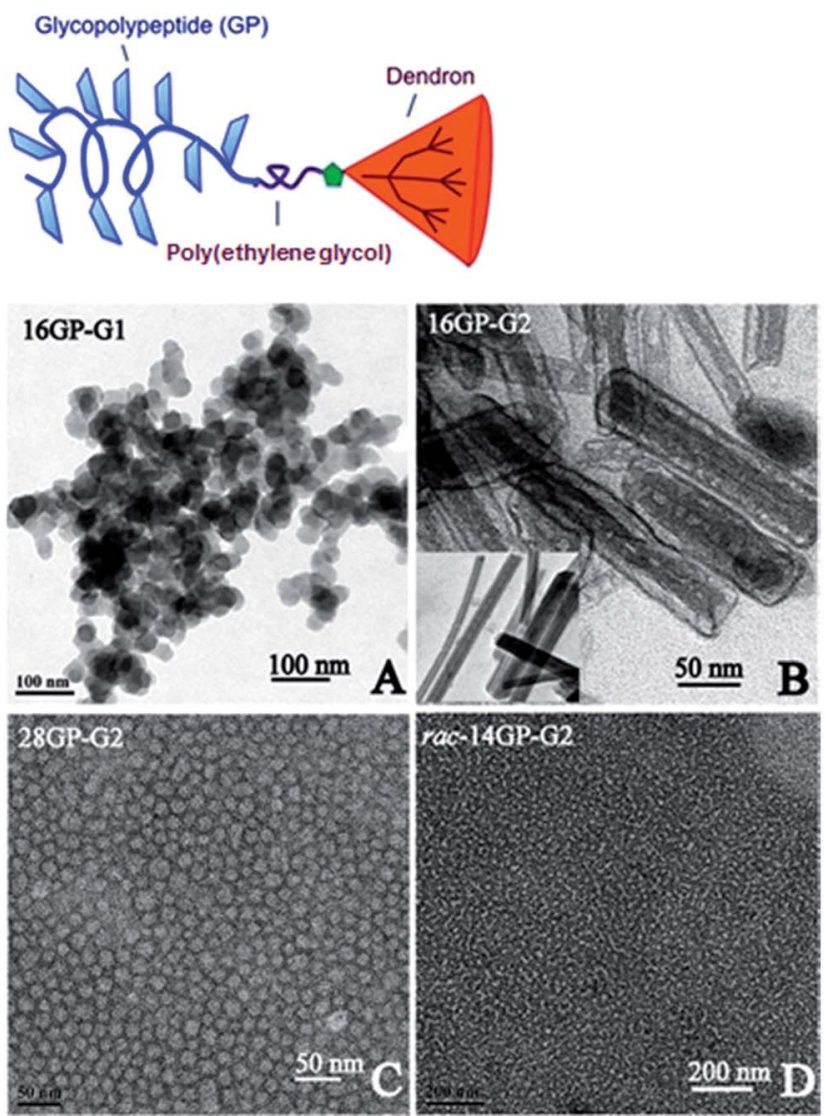

Fig. 4 (Top) Schematic representation of glycopolypeptide-dendron $\left(\mathrm{GP}_{n}-\mathrm{Gx}\right.$ ) conjugates ( $n$ : average number of repeat units, $x$ : dendron generation). (Bottom) Transmission electron micrographs of (A) $\mathrm{GP}_{16}-$ G1, (B) $\mathrm{GP}_{16}-\mathrm{G} 2$, (C) $\mathrm{GP}_{28}-\mathrm{G} 2$, and (D) racGP $14-\mathrm{G} 2$ in water (negatively stained with uranyl acetate). Reprinted with permission from ref. 60, copyright (2012) American Chemical Society.

\section{Drug delivery}

Just one study has been reported on the use of glycopolypeptide block copolymers, more on glycopeptide dendrimers, ${ }^{63,64}$ for targeted drug delivery.
Feng et al. used the glycopolypeptide particles of glycosylated poly(L-lysine)-block-poly(tetrahydrofuran)-block-poly(Llysine) for encapsulation and in vitro release of doxorubicin. ${ }^{52}$ The drug-loaded particles were spherical in shape and 100$150 \mathrm{~nm}$ in diameter. The doxorubicin release rate was found to be stable for about $10-15 \mathrm{~h}$ after an initial rapid release (about 50\% within first $2 \mathrm{~h}$ ) and was faster in an acidic than in a neutral environment. Additionally, the introduction of sugar improved the biocompatibility of the poly(L-lysine) segments.

\section{Summary and outlook}

The developments of new synthetic techniques and procedures toward glycopolypeptides through amino acid $N$-carboxyanhydride polymerization have enabled the preparation of a large number of new bioinspired functional polymer materials. Most of the synthesis studies and developments have been published after 2010, hence just a few reports exist yet on the advanced properties of glycopolypeptides, for instance stimuli-responsive solution behavior and self-assembly, or usage in biomedical applications.

Glycopolypeptides, to our opinion, have immense potentials as bioinspired functional and structural materials, which have little been explored so far. Yet all research efforts are focused on multi-responsive glycopolypeptides and selfassemblies for advanced applications in biomedicine or life science, especially for targeted drug delivery and tissue engineering. Other usages of glycopolypeptides, like for instance fabrication of bulk materials and films with higher-order hierarchical structuring in a water-based process, have not been shown. Glycopolypeptides could be good candidates to mimic the natural hierarchical structures of collagens, $\alpha$ keratins (based on helical glycopolypeptides), and $\beta$-keratins (based on $\beta$-sheet fibrous structures, which are unknown for glycopolypeptides yet) and open new avenues in materials science. 


\section{Acknowledgements}

This work was supported by the Max Planck Society and the German Research Foundation within the IUPAC Transnational Pilot Call in Polymer Chemistry.

\section{References}

1 H. Schlaad and M. Antonietti, Eur. Phys. J. E, 2003, 10, 17-23. 2 H. G. Börner and H. Schlaad, Soft Matter, 2007, 3, 394-408. 3 http://en.wikipedia.org/wiki/Glycopolymer.

4 H. P. Panzer and R. L. Whistler, Chem. Eng. News, 1959, 37, 41.

5 T. P. Bird, W. A. P. Black, E. T. Dewar and D. Rutherford, Chem. Ind., 1960, 1331-1332.

6 S. Kimura and M. Imoto, Makromol. Chem., 1961, 50, 155160.

7 V. Ladmiral, E. Melia and D. M. Haddleton, Eur. Polym. J., 2004, 40, 431-449.

8 S. R. S. Ting, G. Chen and M. H. Stenzel, Polym. Chem., 2010, 1, 1392-1412.

9 A. M. Eissa and N. R. Cameron, Adv. Polym. Sci., 2013, 253, 71-114.

10 Y. Yeh and R. E. Feeney, Chem. Rev., 1996, 96, 601-618.

11 C. R. Bertozzi and L. L. Kiessling, Science, 2001, 291, 23572364.

12 R. A. Dwek, Chem. Rev., 1996, 96, 683-720.

13 M. R. Pratt and C. R. Bertozzi, Chem. Soc. Rev., 2005, 34, 5868.

14 T. Buskas, S. Ingale and G.-J. Boons, Glycobiology, 2006, 16, 113R-136R.

15 C. W. Cairo, J. E. Gestwicki, M. Kanai and L. L. Kiessling, J. Am. Chem. Soc., 2002, 124, 1615-1619.

16 J. E. Gestwicki, C. W. Cairo, L. E. Strong, K. A. Oetjen and L. L. Kiessling, J. Am. Chem. Soc., 2002, 124, 14922-14933.

17 K. Aoi, K. Tsutsumiuchi and M. Okada, Macromolecules, 1994, 27, 875-877.

18 J. Huang and A. Heise, Chem. Soc. Rev., 2013, 42, 7373-7390.

19 C. Bonduelle and S. Lecommandoux, Biomacromolecules, 2013, 14, 2973-2983.

20 J. R. Kramer and T. J. Deming, Polym. Chem., 2014, 5, 671682.

21 M. A. Quadir, M. Martin and P. T. Hammond, Chem. Mater., 2014, 26, 461-476.

22 H. Lu, J. Wang, Z. Song, L. Yin, Y. Zhang, H. Tang, C. Tu, Y. Lin and J. Cheng, Chem. Commun., 2014, 50, 139-155.

23 E. Rüde, O. Westphal, E. Hurwitz, S. Fuchs and M. Sela, Immunochemistry, 1966, 3, 137-151.

24 J. R. Kramer and T. J. Deming, Biomacromolecules, 2010, 11, 3668-3672.

25 J. R. Kramer and T. J. Deming, J. Am. Chem. Soc., 2010, 132, 15068-15071.

26 D. Pati, A. Y. Shaikh, S. Hotha and S. S. Gupta, Polym. Chem., 2011, 2, 805-811.

27 D. Pati, A. Y. Shaikh, S. Das, P. K. Nareddy, M. J. Swamy, S. Hotha and S. S. Gupta, Biomacromolecules, 2012, 13, 1287-1295.
28 S. Das, D. Pati, N. Tiwari, A. Nisal and S. S. Gupta, Biomacromolecules, 2012, 13, 3695-3702.

29 T. Stöhr, A. R. Blaudszun, U. Steinfeld and G. Wenz, Polym. Chem., 2011, 2, 2239-2248.

30 J. R. Kramer and T. J. Deming, J. Am. Chem. Soc., 2012, 134, 4112-4115.

31 M. I. Gibson, G. J. Hunt and N. R. Cameron, Org. Biomol. Chem., 2007, 5, 2756-2757.

32 H. C. Kolb, M. G. Finn and K. B. Sharpless, Angew. Chem., Int. Ed., 2001, 40, 2004-2021.

33 J.-F. Lutz and H. Schlaad, Polymer, 2008, 49, 817-824.

34 C. R. Becer, R. Hoogenboom and U. S. Schubert, Angew. Chem., Int. Ed., 2009, 48, 4900-4908.

35 C. R. Becer, Macromol. Rapid Commun., 2012, 33, 742-752.

36 H. Tang and D. Zhang, Biomacromolecules, 2010, 11, 1585-1592.

37 H. Tang and D. Zhang, Polym. Chem., 2011, 2, 1542-1551.

38 C. Xiao, C. Zhao, P. He, Z. Tang, X. Chen and X. Jing, Macromol. Rapid Commun., 2010, 31, 991-997.

39 J. Huang, G. Habraken, F. Audouin and A. Heise, Macromolecules, 2010, 43, 6050-6057.

40 J. Huang, C. Bonduelle, J. Thévenot, S. Lecommandoux and A. Heise, J. Am. Chem. Soc., 2012, 134, 119-122.

41 C. Bonduelle, J. Huang, E. Ibarboure, A. Heise and S. Lecommandoux, Chem. Commun., 2012, 48, 8353-8355.

42 J. Sun and H. Schlaad, Macromolecules, 2010, 43, 4445-4448.

43 N. Franz and H.-A. Klok, Macromol. Chem. Phys., 2010, 211, 809-820.

44 J. W. Robinson and H. Schlaad, Chem. Commun., 2012, 48, 7835-7837.

45 K.-S. Krannig and H. Schlaad, J. Am. Chem. Soc., 2012, 134, 18542-18545.

46 K.-S. Krannig, J. Huang, A. Heise and H. Schlaad, Polym. Chem., 2013, 4, 3981-3986.

47 J. R. Kramer and T. J. Deming, Biomacromolecules, 2012, 13, 1719-1723.

48 J. R. Kramer and T. J. Deming, Chem. Commun., 2013, 49, 5144-5146.

49 X. X. Zeng, T. Murata, H. Kawagishi, T. Usui and K. Kobayashi, Biosci., Biotechnol., Biochem., 1998, 62, 1171-1178.

50 R. Mildner and H. Menzel, J. Polym. Sci., Part A: Polym. Chem., 2013, 51, 3925-3931.

51 Y. Wang and K. L. Kiick, J. Am. Chem. Soc., 2005, 127, 1639216393.

52 Z. Tian, M. Wang, A. Y. Zhang and Z. G. Feng, Polymer, 2008, 49, 446-454.

53 N. Lupu-Lotan, A. Yaron, A. Berger and M. Sela, Biopolymers, 1965, 3, 625-655.

54 K.-S. Krannig, J. Sun and H. Schlaad, Biomacromolecules, 2014, 15, 978-984.

55 H. Kukula, H. Schlaad, M. Antonietti and S. Förster, J. Am. Chem. Soc., 2002, 124, 1658-1663.

56 R. Sigel, M. Losik and H. Schlaad, Langmuir, 2007, 23, 71967199.

57 H. Lu, J. Wang, Y. G. Bai, J. W. Lang, S. Y. Liu, Y. Lin and J. J. Cheng, Nat. Commun., 2011, 2, 206.

58 M. Huo, J. Yuan, L. Tao and Y. Wei, Polym. Chem., 2014, 5, 1519-1528. 
59 J. R. Kramer, A. R. Rodriguez, U.-J. Choe, D. T. Kamei and T. J. Deming, Soft Matter, 2013, 9, 3389-3395.

60 D. Pati, N. Kalva, S. Das, G. Kumaraswamy, S. S. Gupta and A. V. Ambade, J. Am. Chem. Soc., 2012, 134, 7796-7802.

61 M. Ambrosi, N. R. Cameron and B. G. Davis, Org. Biomol. Chem., 2005, 3, 1593-1608.
62 X. X. Zeng, T. Murata, H. Kawagishi, T. Usui and K. Kobayashi, Carbohydr. Res., 1998, 312, 209-217.

63 A. S. Zarena and S. Gopal, Mini-Rev. Med. Chem., 2013, 13, 1448-1461.

64 T. Darbre and J. L. Reymond, Curr. Top. Med. Chem., 2008, 8, 1286-1293. 